IOS Press

\title{
Early hyaluronate injection improves quality of life but not neural recovery in unilateral vocal fold paralysis: An open-label randomized controlled study
}

\author{
Yu-Cheng Pei ${ }^{\mathrm{a}, \mathrm{b}, \mathrm{c}, 1}$, Tuan-Jen Fang ${ }^{\mathrm{b}, \mathrm{d}, *, 1}$, Li-Jen Hsin ${ }^{\mathrm{b}, \mathrm{d}}$, Hsueh-Yu Li ${ }^{\mathrm{b}, \mathrm{d}}$ and Alice MK Wong ${ }^{\mathrm{a}, \mathrm{b}, \mathrm{c}}$ \\ ${ }^{a}$ Department of Physical Medicine and Rehabilitation, Chang Gung Memorial Hospital at Linkou, Taoyuan, Taiwan \\ ${ }^{\mathrm{b}}$ School of Medicine, Chang Gung University, Taoyuan, Taiwan \\ ${ }^{\mathrm{c}}$ Healthy Aging Research Center, Chang Gung University, Taoyuan, Taiwan \\ ${ }^{\mathrm{d}}$ Department of Otolaryngology, Chang Gung Memorial Hospital at Linkou, Taoyuan, Taiwan
}

\begin{abstract}
.
Purpose: To investigate the neurologic and functional effect of intracordal hyaluronate injections in acute unilateral vocal fold paralysis (UVFP) in a randomized controlled trial.

Methods: In this open-label, randomized controlled study, 29 patients with UVFP were recruited within 6 months of their first outpatient visit and were randomized to receive either single hyaluronate injection (HI group) or conservative management (CM group). Quantitative laryngeal electromyography, videolaryngostroboscopy, UVFP-related quality of life (Voice Outcomes Survey, VOS), laboratory voice analysis, and health-related quality of life (SF-36) were evaluated at baseline, and at 1, 3 and 6 months post-injection in the HI group, and at baseline and 6 months in the CM group.

Results: Improvements in most quality of life domains and other assessments were comparable between the HI and CM groups; however, the HI group had a greater improvement in the mental health domain of quality of life at the end of follow-up. Conclusions: Early hyaluronate injection cannot improve nerve regeneration but can result in long-lasting improvements in patients' psychosocial well-being, thus highlighting the importance of early intervention for patients with UVFP.
\end{abstract}

Keywords: Unilateral vocal fold paralysis, hyaluronate, injection laryngoplasty, glottal gap, quality of life, laryngeal electromyography

Clinical trial protocol: ClinicalTrials.gov Identifier: NCT02163772

\section{Introduction}

Unilateral vocal fold paralysis (UVFP) manifesting with dysphonia is a common complication of thyroid, cervical spine, esophagus, and lung surgeries

\footnotetext{
${ }^{1}$ Equal contribution as the first author.

*Corresponding author: Tuan-Jen Fang, MD, FICS, Department of Otolaryngology, Chang Gung Memorial Hospital at Linkou, 5 Fushing St., Taoyuan 333, Taiwan. Tel.: +886 33281200/Ext. 3971; Fax: +886 33979361; E-mail: fang3109@adm.cgmh.org.tw.
}

(Ko, 2009; Pei, 2014). Overall, approximately 50\% of cases of UVFP are caused by surgery (Ko, 2009; Rosenthal, 2007; Yumoto, 2002). The development of office-based injection laryngoplasty techniques (Blitzer, 2009) with safe and effective fillers such as hyaluronate (Song, 2010) allows for early intervention, and could be considered as standard therapy for patients with surgically-induced UVFP. However, evidence is needed to characterize the short- and longterm benefits of early-injection laryngoplasty. In early 
injection laryngoplasty, the injected substance is presumed to be metabolized during the initial 1-3 months after injection (Lau, 2010). In this manner, substances, such as hyaluronate, transiently expand the tissue volume. However, such injections could also have a long-lasting effect if given during the early phase of UVFP, through promoting rehabilitation in patients with a large glottal gap (Fang, 2014). We conducted an open-label, randomized controlled study to evaluate the effects of a single hyaluronate injection during the early phase of UVFP, and characterized the therapeutic effects after 6 months follow-up. The prospective, randomized design allowed both the immediate and long-term impacts of hyaluronate injection on acute UVFP to be assessed. We hypothesized that although the physiological effects of early hyaluronate injection may have disappeared by 6 months follow-up, other effects might be longer lasting.

\section{Materials and methods}

\subsection{Patients}

The study was approved by the Ethics Committee and the Institutional Review Board of Chang Gung Medical Foundation. Written informed consent was obtained from each participant prior to recruitment. Patients with UVFP manifesting with dysphonia diagnosed within 6 months from symptom onset were invited to participate. The diagnosis of UVFP was confirmed by both laryngoscopy and laryngeal electromyography (LEMG). Exclusion criteria were a history of prior vocal fold palsy, inability to cooperate with evaluations, or lack of evidence of denervation changes in the thyroarytenoid muscle in the involved side. Patients were randomized and allocated to one of two groups using a computer program (Fig. 1). One group underwent office-based hyaluronate (Restylane, Q-Med AB, Uppsala, Sweden) injection(HIgroup) through the cricothyroid membrane and the other group received conventional conservative management(CM group), following the observational watch-and-wait policy. Both the $\mathrm{HI}$ and $\mathrm{CM}$ groups received vocal hygiene and swallowing education at each outpatient visit.

\subsection{Assessments}

At each assessment, patients were monitored by quantitative LEMG, videolaryngostroboscopy, Voice

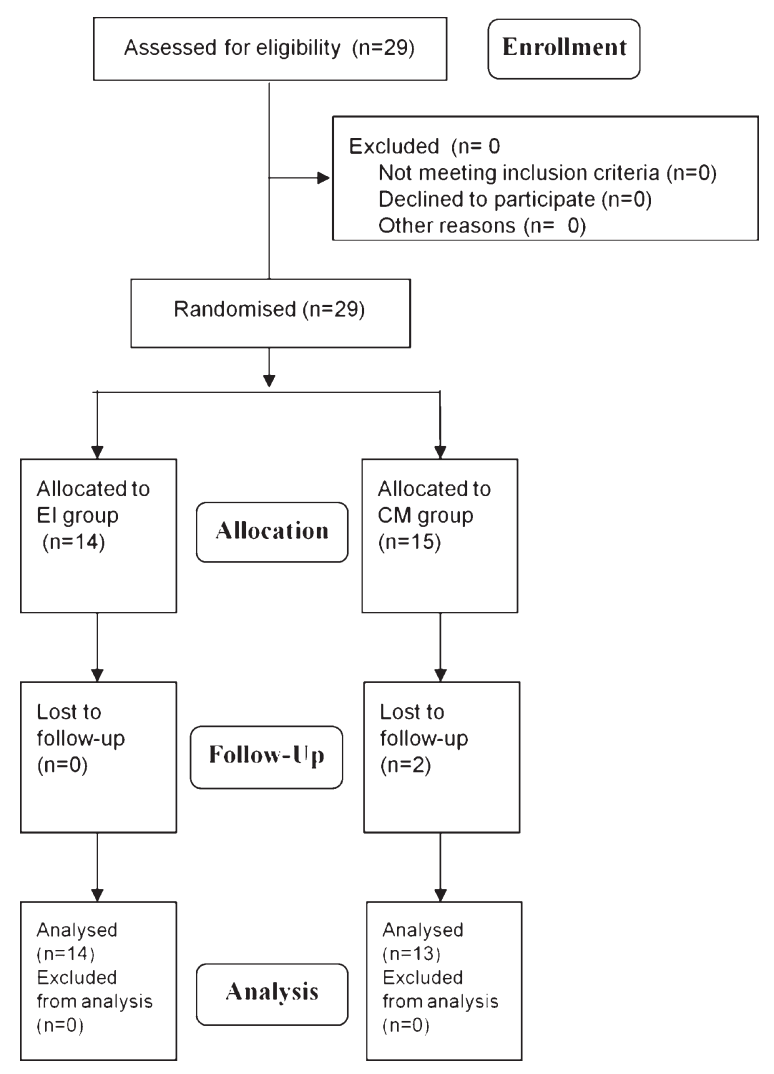

Fig. 1. Study flowchart.

Outcome Survey (VOS), laboratory voice analysis, and SF-36 health-related quality of life. The HI group received assessments at baseline and at 1, 3 and 6 months post-injection, while the CM group at baseline and after 6 months. Figure 1 illustrates a flowchart for the present study.

\subsubsection{LEMG examination}

LEMG and quantitative LEMG were conducted as described previously (Pei, 2014). LEMG signals were obtained using a concentric needle electrode with the surface ground electrode adhered to the forehead. EMG signals were observed on bilateral thyroarytenoid-lateral cricoarytenoid (TA-LCA) muscle complexes and cricothyroid (CT) muscles. With the concentric needle electrode in the TA-LCA muscle complex, the patient was asked to produce three series of "e"s at three different intensities (low, moderate and highest possible), with each "e" lasting at least $400 \mathrm{~ms}$ and each inter-"e" interval lasting about $200 \mathrm{~ms}$ 
(Pei, 2014). To evaluate CT function, the patients were asked to produce a glissando upward "e" at normal loudness.

We initially observed insertional activity and spontaneous activity for each test. We then performed semiquantitative motor unit analysis and recruitment analysis, specifically when the rise time of a motor unit action potential was $<0.6 \mathrm{~ms}$, indicating a close proximity to the recorded motor unit. A Nicolet Viking Select (Cardinal Health, OH, USA) was used with its band-pass filter set between $20 \mathrm{~Hz}$ and $10 \mathrm{kHz}$. An abnormal LEMG was defined as the existence of spontaneous activities (such as fibrillation, positive sharp wave, or complex repetitive discharge), $>30 \%$ polyphasia, or decreased interference pattern (reduced, discrete, or no interference pattern). Motor unit recruitment tracings were recorded with sweep speeds of $10 \mathrm{~ms}$ per division and a gain of $200 \mu \mathrm{V}$ per division.

\subsubsection{Quantitative LEMG analysis}

We first binned the raw EMG data in each 200-ms epoch (Pei, 2014). An automatic algorithm was used to localize the timing of each turn and its amplitude. Specifically, a turn was defined by a change in polarity with an amplitude of at least $100 \mu \mathrm{V}$ before and after the change, to exclude peaks inherent to noise. Turn frequency was computed for each epoch as the number of turns divided by epoch duration, and mean turn amplitude was computed as the mean of the absolute turn-amplitude values. For the TA-LCA muscle complex, turn frequencies were averaged for epochs, with turn frequencies among the top three epochs to yield the peak turn frequency.

\subsubsection{Laryngeal configuration: The normalized glottal gap area}

An image of the glottis was captured during videolaryngoscopy while the patient vocalized /eee/ at modal pitch and regular loudness. The images showing the narrowest vocal slit from several phonatory cycles were used in the calculation. The normalized glottal gap area (NGGA) was analyzed using image-processing computer software (Image J $1.44 \mathrm{p}$, National Institutes of Health, Bethesda, MD, USA) using the method proposed by Omori et al. (Omori, 1996) The traced glottal gap area was calculated and expressed in square pixels. Membranous vocal-fold length was measured from the point of the anterior commissure to the point of the tip of the vocal process and was expressed in linear pixels. The NGGA was computed using the following Equation (1):

$$
\begin{aligned}
\text { NGGA = } & \text { (narrowest glottal gap area/membranous } \\
& \text { vocal fold length })^{2} \times 100 \text { units }
\end{aligned}
$$

\subsubsection{Voice outcome survey}

The VOS originally developed by Gliklich et al. (Gliklich, 1999) is a five-item survey that evaluates the physical and social problems associated with UVCP on a Likert scale. It has been translated into Mandarin Chinese following a standard survey-validation process by the present research team (Fang, 2007). The survey items and total scores were normalized on a 0 (worst) to 100 (best) scale, based on published algorithms.

\subsubsection{Laboratory voice analysis}

In a sound-insulated room, the patient was asked to read a standard passage and a sustained vowel at a conversational pitch and loudness. The patient's voice was captured using a unidirectional dynamic microphone (Shure SM48; Shure Brothers Inc., Agua Prieta, Mexico) with a mouth-to-microphone distance of $10 \mathrm{~cm}$ and an off-axis angle of $45^{\circ}$. Voice was then recorded and sampled using a voice-analysis computer program (Computerized Speech Lab model 4300B, version 5.05; Kay Elemetrics Corp., Lincoln Park, IL, USA) with a sampling rate of $25.6 \mathrm{kHz}$ and 16-bit quantization. Fundamental frequency, jitter (perturbation of frequency), shimmer (perturbation of amplitude), and harmonic-to-noise ratio were tabulated from the recorded voice. The maximal phonation time represented the duration that a patient could sustain a vowel /a/. The SZ ratio is the ratio of the duration of a /s/ to a /z/, which represents vocal cord control. The ideal value is close to 1 .

\subsubsection{Health-related quality of life (SF-36)}

The SF-36 questionnaire was used to evaluate health-related quality of life. The SF-36 consisted of eight scaled scores for vitality, physical functioning, bodily pain, general health perceptions, physical-role functioning, emotional-role functioning, social functioning, and mental health. Each score is tabulated according to the published algorithms on a scale of 0 (maximal disability) to 100 (no disability). The recall period of the SF-36 is 4 weeks. The International Quality of Life Assessment Standard Taiwan version 1.0 was used (Lu, 2003; Tseng, 2003). 


\subsection{Intracordal hyaluronate injection (Appendix: Video clip)}

Patients in the HI group received intracordal hyaluronate injection within 6 months of their initial symptoms. Before injection, the nasal mucosa was anesthetized by spraying with $2 \%$ lidocaine with neo-synephrine $1: 100,000$, and the oral cavity was sprayed with $10 \%$ lidocaine. Patients were seated upright with their neck extended. After the subject felt numbing of their throat, a distal-chip laryngoscope (Laryngoscope: ENF TYPE V2; Platform: EVIS Exera II; Olympus Optical Co, Ltd, Tokyo, Japan) was inserted transnasally, allowing the glottis to be viewed on a monitor. A 23- or 25-gauge needle was inserted through the cricothyroid membrane into the vocal folds and approximately $0.5 \mathrm{ml}$ of $2 \%$ lidocaine was pushed into the laryngo-trachea. A needle containing hyaluronate was first placed on the paramidline point at the level of the cricothyroid junction. After the needle passed through the cricothyroid membrane, it was moved upward gently submucosally to confirm the location of the tip, by moving it forward and backward by a short distance (Fig. 2). It is crucial to avoid placing the needle tip in the airway or in Reinke's space in the

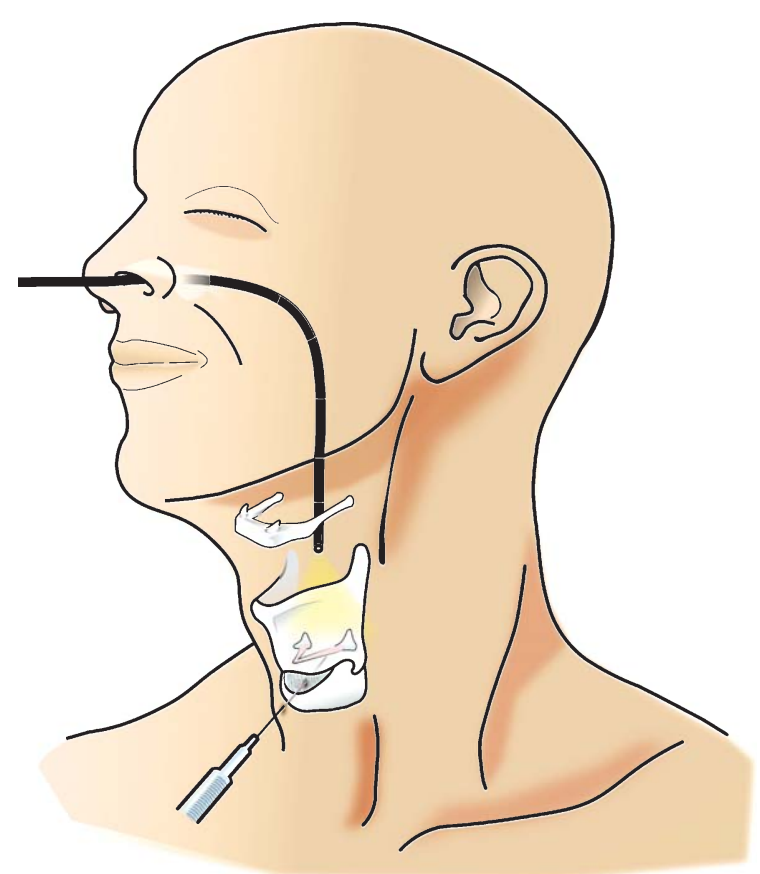

Fig. 2. Trans-cricothyroid membrane injection of hyaluronate under illumination and monitoring by the flexible laryngoscopy. vocal fold. After the location was confirmed, an adequate amount of hyaluronate was slowly pushed into the vocal folds. The patient was then asked to project their voice at the end of the injection to confirm vocal fold position and voice satisfaction (Fig. 3).

\subsection{Statistical analysis}

The data were analyzed using SPSS (SPSS Inc., Chicago, IL, USA). Comparisons of baseline assessments between the two groups were performed using Student's $t$-tests for parametric data and $\chi^{2}$ tests for categorical data. Comparisons of changes in parameters over time between the two groups were conducted using repeated measures ANOVA. The $\alpha$ value was defined as 0.05 .

\section{Results}

Among the 29 dysphonic patients recruited, one died and one refused follow-up, leaving data for 27 (14 in the HI group and 13 in the CM group) to be analyzed (Fig. 1 and Table 1). Patient demographics, etiology of UVFP, NGGA, peak turn frequency, voice, laboratory voice analysis, and quality of life at initial assessment are listed in Table 1. Regarding the etiology of UVFP, 23 of the 27 (85\%) patients developed UVFP as a result of surgical complications, and the remaining four had other causes. There were no significant differences in sex, age, NGGA, peak turn frequency, VOS, most parameters of laboratory voice analysis, and SF36 domains between the two groups ( $p>0.05)$, though maximum phonation time was longer in the HI group ( $p=0.03$ ) compared with the CM group.

None of the patients in the HI group experienced complications of hyaluronate injection. Table 2 shows the results of assessments performed at baseline and at 6-months follow-up. Patients in both groups showed substantial improvements in NGGA, peak turn frequency, VOS, and parameters of laboratory voice analysis (except fundamental frequency) at 6-months follow-up. Interaction effect of repeated-measures ANOVA showed that there were no differences in improvements between the two groups in terms of NGGA, peak turn frequency, or VOS parameters in laboratory voice analysis $(p>0.05)$ (Table 2$)$.

Regarding quality of life, patients in the HI group showed a significantly higher improvement in mental health (interaction effect, $p=0.03$ ) and a borderline 


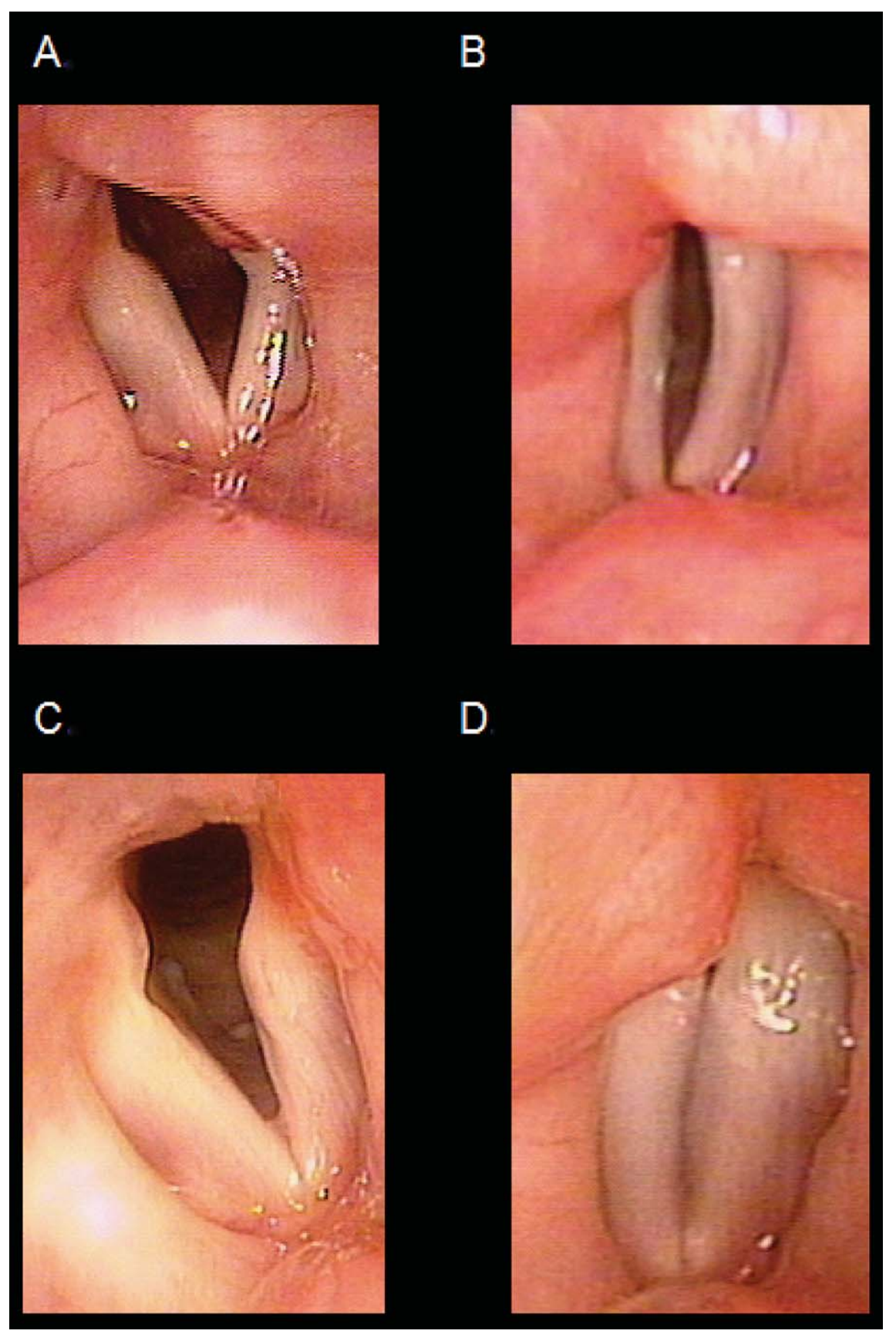

Fig. 3. Vocal fold position before and after an office-based hyaluronate injection for a patient with left UVFP. A. Before injection (breathing). B. Before injection (phonation). C. After injection (breathing). D. After injection (phonation).

higher improvement in social role functioning (interaction effect, $p=0.06$ ) compared with CM patients. However, improvements in other quality of life domains were comparable between the two groups (interaction effect, $p>0.05$ ).
We characterized the temporal dynamics of the effects induced by the hyaluronate injections (Fig. 4). Most measurements showed immediate improvements after hyaluronate injection, with persisting effect at the 3- or 6-month follow-up. This pattern was observed 

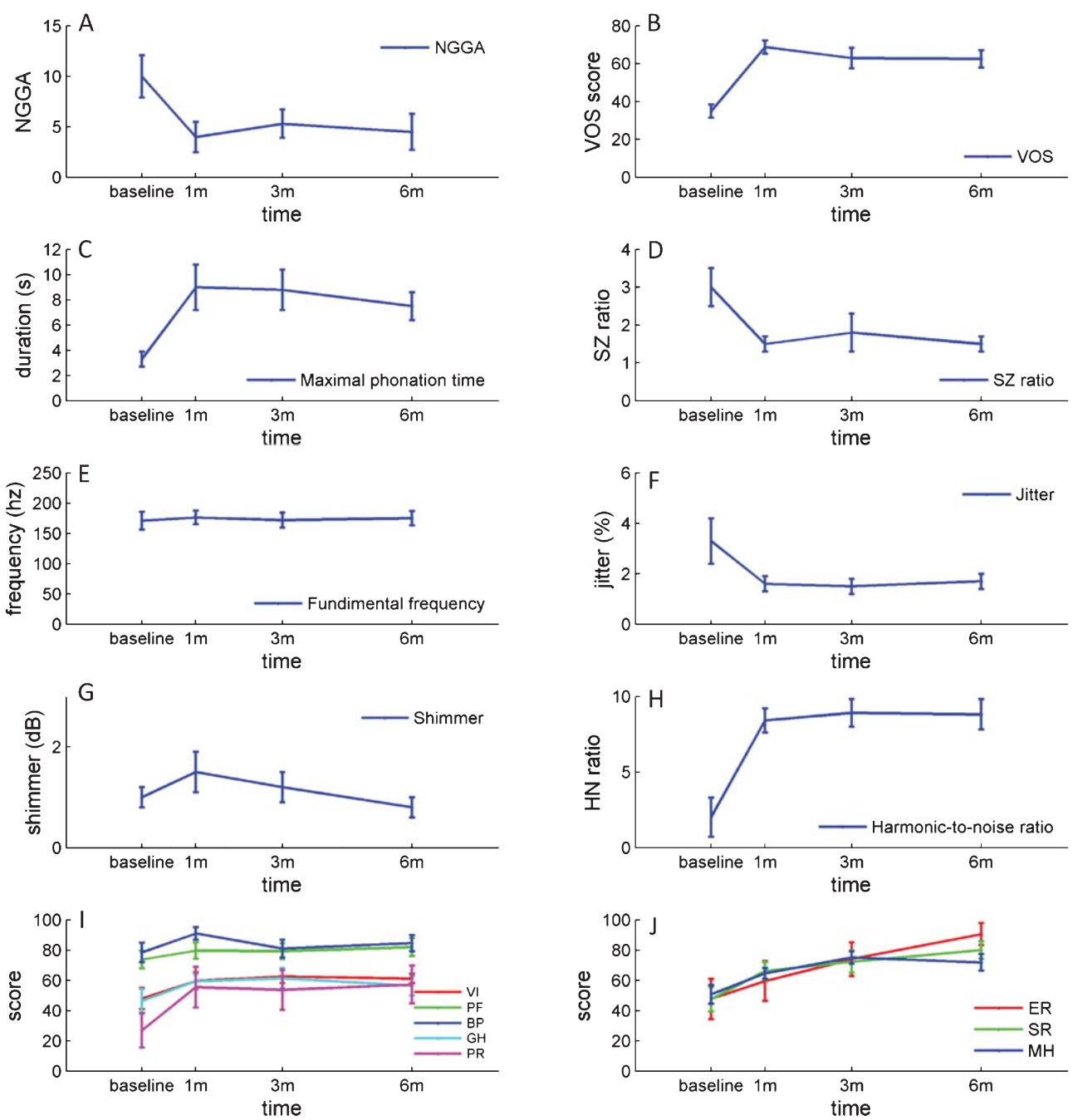

Fig. 4. Temporal dynamics of NGGAs, VOS, laboratory voice analysis, and quality of life in the treatment group at baseline and at 1,3 and 6 months post-injection. A. Closed and open NGGAs. B. VOS. C. Maximal phonation time. D. SZ ratio. E. Fundamental frequency. F. Jitter. G. Shimmer. H. Harmonic-to-noise ratio. I. Quality of life domains that improved abruptly after injection and remained constant thereafter. J. Quality of life domains demonstrating gradual changes during long-term follow-up. VI vitality, $P F$ physical functioning, $B P$ bodily pain, $G H$ general health perceptions, $P R$ physical role functioning, $E R$ emotional role functioning, $S R$ social role functioning, $M H$ mental health.

for NGGA (Fig. 4A), VOS (Fig. 4B), maximal phonation time (Fig. 4C), SZ ratio (Fig. 4D), jitter (Fig. 4F), shimmer (Fig. 4G), harmonic-to-noise ratio (Fig. 4H), and quality of life domains of vitality, physical functioning, bodily pain, general health perceptions, and physical role functioning (Fig. 4I). In contrast, fundamental frequency was unaffected by hyaluronate injection and remained constant throughout the followup assessments (Fig. 4E). Interestingly, the quality of life domains of emotional role functioning, social role functioning, and mental health showed continuous improvements, even months after the injection
(Fig. 4J), indicating a long-lasting effect of hyaluronate injection on these psychosocial domains.

\section{Discussion}

Recurrent laryngeal nerve injury manifesting with UVFP is a fairly common surgical complication. Patients with recurrent laryngeal nerve injury suffer from breathy voice, swallowing disturbances, and poor breathing control. Although surgical techniques such as thyroplasty and lipoinjection have proven effective 
Table 1

Patient demographics and baseline measurements

\begin{tabular}{|c|c|c|c|}
\hline & HI group & $\mathrm{CM}$ group & $p$ value \\
\hline $\operatorname{Sex}(M / F)$ & $7 / 7$ & $6 / 7$ & 0.84 \\
\hline Age (year) & $49.4 \pm 3.3$ & $52.2 \pm 4.4$ & 0.61 \\
\hline \multicolumn{4}{|l|}{ Etiology } \\
\hline Lung/mediastinum surgery & 3 & 2 & \\
\hline Esophageal surgery & 2 & 1 & \\
\hline Carotid body tumor resection & 0 & 1 & \\
\hline Thyroidectomy/parathyroidectomy & 6 & 6 & \\
\hline Skull base surgery & 2 & 0 & \\
\hline Cervical spine surgery & 0 & 0 & \\
\hline Idiopathic & 1 & 3 & \\
\hline NGGA & $10.0 \pm 2.1$ & $11.4 \pm 3.7$ & 0.74 \\
\hline \multicolumn{4}{|l|}{ Recruitment analysis } \\
\hline Lesion side (turn/s) & $261.1 \pm 52.4$ & $317.9 \pm 77.7$ & 0.55 \\
\hline Normal side (turn/s) & $884.7 \pm 127.6$ & $674.4 \pm 81.5$ & 0.18 \\
\hline Turn ratio $0.46 \pm 0.11$ & $0.52 \pm 0.12$ & 0.71 & \\
\hline \multicolumn{4}{|l|}{ Laboratory voice analysis } \\
\hline Maximum phonation time (s) & $3.3 \pm 0.6$ & $5.9 \pm 1.0$ & $0.03^{*}$ \\
\hline $\mathrm{SZ}$ ratio & $3.0 \pm 0.5$ & $3.2 \pm 1.3$ & 0.92 \\
\hline Fundamental frequency $(\mathrm{Hz})$ & $171 \pm 14.7$ & $181.5 \pm 9.1$ & 0.55 \\
\hline Jitter $(\%)$ & $3.3 \pm 0.9$ & $2.9 \pm 0.4$ & 0.66 \\
\hline Shimmer $(\mathrm{dB})$ & $1.0 \pm 0.2$ & $1.4 \pm 0.3$ & 0.31 \\
\hline Harmonic-to-noise ratio & $1.9 \pm 1.3$ & $2.6 \pm 2.2$ & 0.82 \\
\hline \multicolumn{4}{|l|}{ Quality of life } \\
\hline Vitality & $47.9 \pm 7.0$ & $57.7 \pm 7.0$ & 0.33 \\
\hline Physical functioning & $73.6 \pm 5.8$ & $88.1 \pm 3.8$ & 0.05 \\
\hline Bodily pain & $78.4 \pm 6.4$ & $83.2 \pm 7.5$ & 0.63 \\
\hline General health perceptions & $46.4 \pm 7.8$ & $55.8 \pm 5.3$ & 0.34 \\
\hline Physical role functioning & $26.8 \pm 11.3$ & $44.2 \pm 13.9$ & 0.34 \\
\hline Emotional role functioning & $47.6 \pm 13.4$ & $79.5 \pm 11.0$ & 0.08 \\
\hline Social functioning & $47.4 \pm 8.0$ & $65.5 \pm 8.3$ & 0.13 \\
\hline Mental health & $50.6 \pm 6.2$ & $63.4 \pm 6.9$ & 0.18 \\
\hline
\end{tabular}

Data presented as mean \pm standard error of mean, ${ }^{*} p<0.05 . H I$ hyaluronate injection, $C M$ conservative management.

in managing UVFP, it is recommended that these definitive therapies be postponed for 9-12 months to allow for the possibility of natural recovery within this period (Woodson, 2007). Most patients only receive conservative observation during this wait-andsee period and the immediate UVFP-related symptoms may subsequently persist and impact on their quality of life. Development of the distal chip laryngoscope and novel injectable materials have increased the popularity of injection laryngoplasty with temporary substances during the early phase after recurrent laryngeal nerve injury, but the effectiveness of this approach remains to be elucidated.

The present study showed that a single hyaluronate injection during the early phase of UVFP improved mental health at 6-months follow-up. However, hyaluronate injection did not alter motor unit recruitment, glottal gap, voice or the results of laboratory voice analysis at 6-months follow-up. The results indicate that a single hyaluronate injection may have a long-term effect on the patient's psychosocial wellbeing without affecting neural regeneration, vocal cord position, or other functional outcomes.

Previous investigators proposed that injection of temporary agents during the early phase of UVFP may facilitate functional recovery. Friedman et al. hypothesized that long-term voice improvement after temporary agent injection may result from vibro-tactile stimulation induced by physical contact of mobile counter cords, i.e., early temporary agent injection increased sensory stimulation of the paralyzed vocal cords, resulting in better vocal-fold positioning (Friedman, 2010). Prendes et al. (Prendes, 2012) evaluated voice in UVFP patients using the Consensus AuditoryPerceptual Evaluation of Voice and found that voice at the final assessment was better in patients who received temporary agent injection. However, this was in contrast to our study, which showed comparable voice improvements in the two groups. This discrepancy could be explained by differences between the 
Table 2

Changes in measurements between baseline and 6-months follow-up in the two groups

\begin{tabular}{|c|c|c|c|c|c|c|}
\hline & \multicolumn{2}{|c|}{ HI group } & \multicolumn{2}{|c|}{$\mathrm{CM}$ group } & \multirow[t]{2}{*}{$F$ value } & \multirow[t]{2}{*}{$p$ value } \\
\hline & baseline & 6 months & baseline & 6 months & & \\
\hline NGGA & $10.0 \pm 2.1$ & $4.5 \pm 1.8$ & $10.7 \pm 3.2$ & $5.0 \pm 1.6$ & 0.08 & 0.78 \\
\hline \multicolumn{7}{|l|}{ Peak turn frequency } \\
\hline Lesion side (turn/s) $\dagger$ & $261.1 \pm 52.4$ & $417.1 \pm 113.4$ & $317.9 \pm 77.7$ & $551.7 \pm 116.3$ & 0.32 & 0.58 \\
\hline Normal side (turn/s) $\dagger$ & $884.7 \pm 127.6$ & $837.4 \pm 112.4$ & $674.4 \pm 81.5$ & $732.6 \pm 97.6$ & 0.39 & 0.54 \\
\hline VOS & $34.9 \pm 3.5$ & $62.6 \pm 4.6$ & $42.1 \pm 4.5$ & $70.0 \pm 4.7$ & & \\
\hline \multicolumn{7}{|l|}{ Laboratory voice analysis } \\
\hline Maximum phonation time (s) & $3.3 \pm 0.6$ & $7.5 \pm 1.1$ & $5.9 \pm 1.0$ & $11.7 \pm 1.9$ & 0.65 & 0.43 \\
\hline SZ ratio & $3.0 \pm 0.5$ & $1.5 \pm 0.2$ & $3.2 \pm 1.3$ & $1.2 \pm 0.1$ & 0.09 & 0.77 \\
\hline Fundamental frequency $(\mathrm{Hz})$ & $171 \pm 14.7$ & $175 \pm 12$ & $181.5 \pm 9.1$ & $193 \pm 17$ & 0.32 & 0.58 \\
\hline Jitter $(\%)$ & $3.3 \pm 0.9$ & $1.7 \pm 0.3$ & $2.9 \pm 0.4$ & $2.1 \pm 0.4$ & 0.68 & 0.42 \\
\hline Shimmer $(\mathrm{dB})$ & $1.0 \pm 0.2$ & $0.8 \pm 0.2$ & $1.4 \pm 0.3$ & $1.4 \pm 0.3$ & 0.26 & 0.62 \\
\hline Harmonic-to-noise ratio (dB) & $1.9 \pm 1.3$ & $8.8 \pm 1.0$ & $2.6 \pm 2.2$ & $6.3 \pm 1.1$ & 0.70 & 0.41 \\
\hline \multicolumn{7}{|l|}{ Quality of life } \\
\hline Vitality & $47.9 \pm 7.0$ & $61.0 \pm 3.1$ & $57.7 \pm 7.0$ & $64.2 \pm 5.5$ & 0.67 & 0.42 \\
\hline Physical functioning & $73.6 \pm 5.8$ & $81.8 \pm 5.9$ & $88.1 \pm 3.8$ & $90.4 \pm 3.6$ & 0.50 & 0.49 \\
\hline Bodily pain & $78.4 \pm 6.4$ & $84.6 \pm 5.4$ & $83.2 \pm 7.5$ & $87.5 \pm 5.6$ & 0.05 & 0.82 \\
\hline General health perceptions & $46.4 \pm 7.8$ & $56.4 \pm 6.4$ & $55.8 \pm 5.3$ & $65.8 \pm 5.7$ & 0 & 1.0 \\
\hline Physical role functioning & $26.8 \pm 11.3$ & $57.1 \pm 12.4$ & $44.2 \pm 13.9$ & $84.6 \pm 8.3$ & 0.23 & 0.64 \\
\hline Emotional role functioning & $47.6 \pm 13.4$ & $90.5 \pm 7.4$ & $79.5 \pm 11.0$ & $82.1 \pm 10.4$ & 4.94 & 0.36 \\
\hline Social role functioning & $47.4 \pm 8.0$ & $79.5 \pm 5.7$ & $65.5 \pm 8.3$ & $76.0 \pm 7.3$ & 3.78 & 0.06 \\
\hline Mental health & $50.6 \pm 6.2$ & $71.7 \pm 5.5$ & $63.4 \pm 6.9$ & $68.0 \pm 5.1$ & 5.75 & $0.02^{*}$ \\
\hline
\end{tabular}

Data presented as mean \pm standard error of mean, ${ }^{*} p<0.05,{ }^{\dagger} \mathrm{n}=10 / 8$ in HI/CM groups, respectively. $H I$ hyaluronate injection, $C M$ conservative management.

two study populations: Prendes et al. recruited patients with a mean disease duration of 10 months, while we recruited patients with a disease duration of less than 6 months. In acute UVFP, voice may recover spontaneously with time, even without recovery of the vocal fold position, and these patients might not have been enrolled in the previous retrospective study because they were symptom-free.

Intracordal injection with a temporary agent in patients with acute UVFP immediately improves their swallowing and voice function (Song, 2010). In the present study, voice and quality of life measurements improved immediately after hyaluronate injection and persisted for 6 months. However, the long-term effect of single injection of a temporary agent remains questionable (Friedman, 2010; Prendes, 2012; Yung, 2011). In a cohort study, Friedman et al. showed that $63 \%$ of patients who received hyaluronate injection within 6 months of symptom onset remained in adequate voice at long-term follow-up (Friedman, 2010). In addition, early hyaluronate injection decreased the need for transcervical medialization laryngoplasty in patients with UVFP (Friedman, 2010; Prendes, 2012). However, no randomized controlled study has addressed this question and no comprehensive evaluation of the effects of hyaluronate injection, involving quantitative recruit- ment analysis, vocal cord position, voice, and quality of life, has yet been made. The discrepancy between the results of the present study and the conclusions drawn from the need for permanent laryngoplasty (Friedman, 2010; Prendes, 2012; Yung, 2011) can be explained by the fact that the decision to perform permanent laryngoplasty is dynamic and multi-factorial, and involves not only the patient's voice function, but also their mental health and interaction with their physician.

The results of the present study indicate that early hyaluronate injection does not change the natural course of nerve regeneration or voice recovery. Woodson suggested that spontaneous motor nerve regeneration occurs after recurrent laryngeal nerve injuries, accompanied by changes in paralyzed vocal cord position and recovery of larynx function(Woodson, 2007). The general consensus is that spontaneous nerve regeneration stops within 9 months from symptom onset, and definitive intervention should therefore start after this time period (Sulica, 2008). Although definitive treatments, such as laryngeal framework surgery or autologous fat injection laryngoplasty, have demonstrated longterm effectiveness (Fang, 2009; Netterville, 1993), the present study supports the use of early hyaluronate injection by demonstrating associated improvements in long-term psychological well-being. This long-term 
effect on mental health may be accounted for by the recovery of voice function during the early phase of UVFP. Specifically, this intervention allows the patient to resume their social role, thus alleviating the impact of voice dysfunction and giving the patient more time to develop a coping strategy.

The present study had several limitations. First, the sample size was small (29 patients), thus limiting its evidence level. Second, although injected hyaluronate is absorbed within 3 months (Lau, 2010), it is possible that residual hyaluronate remained in the vocal cord after this time, thus contributing to its long-term effects. Further studies with a longer follow-up period are needed to address this possibility. Another direction of research is to apply adjunct therapies, such as electrical stimulation, that have been showed to be effective in improving the recovery of function in animal models (Brown, 2013; Monaco, 2013; Wang, 2013).

\section{Conclusion}

Early hyaluronate intracordal injection improved voice and quality of life immediately in patients with UVFP. In comparison with the conventional observation policy, a single early injection of hyaluronate improves mental health at 6-months follow-up. These results suggest that early hyaluronate injection can result in long-lasting improvements in patients' psychosocial well-being, thus highlighting the importance of early intervention for patients with UVFP.

\section{Acknowledgments}

The research was supported by a National Science Council Grant (NSC 100-2314-B-182-021, NSC 1012314-B-182-035, NSC 102-2314-B-182A-080) and Chang Gung Medical Foundation Grant (CMRPG 3B1032). The funder had no role in study design, data collection and analysis, decision to publish, or preparation of the manuscript.

\section{Author contributions}

YCP TJF: Conceived and designed the experiments. YCP TJF: Performed the experiments.

YCP TJF: Contributed materials and analysis tools. YCP TJF LJH HYL AMKW: Wrote the manuscript.

\section{References}

Blitzer, A.B., M.F., \& Ramig, L.O. (2009). Neurologic disorders of the larynx (2nd ed.), New York: Thieme.

Brown, T.J., Pittman, A.L., Monaco, G.N., Benscoter, B.J., Mantravadi, A.V., Akst, L.M., \& Foecking, E.M. (2013). Androgen treatment and recovery of function following recurrent laryngeal nerve injury in the rat. [Research Support, Non-U.S. Gov't]. Restor Neurol Neurosci, 31(2), 169-176. doi:10.3233/RNN-120287

Brünings, W. (1911). Uber eine neue Behandelungs Methodeder Rekurrens-lamung. Verhandl Ver Deutch Laryngol, 19, 93-98.

Clary, M.S., \& Courey, M.S. (2013). Development of procedures and techniques for the office. [Historical Article]. Otolaryngol Clin North Am, 46(1), 1-11. doi: 10.1016/j.otc.2012.08.013

Fang, T.J., Lee, L.A., Wang, C.J., Li, H.Y., \& Chiang, H.C. (2009). Intracordal fat assessment by 3-dimensional imaging after autologous fat injection in patients with thyroidectomy-induced unilateral vocal cord paralysis. [Clinical Trial]. Surgery, 146(1), 82-87. doi: 10.1016/j.surg.2009.02.005

Fang, T.J., Li, H.Y., Gliklich, R.E., Chen, Y.H., \& Wang, P.C. (2007). Assessment of Chinese-version voice outcome survey in patients with unilateral vocal cord paralysis. [Validation Studies]. Otolaryngol Head Neck Surg, 136(5), 752-756. doi: 10.1016/j.otohns.2006.11.048

Fang, T.J., Pei, Y.C., Li, H.Y., Wong, A.M., \& Chiang, H.C. (2014). Glottal gap as an early predictor for permanent laryngoplasty in unilateral vocal fold paralysis. Laryngoscope. doi: 10.1002/lary.24689

Friedman, A.D., Burns, J.A., Heaton, J.T., \& Zeitels, S.M. (2010). Early versus late injection medialization for unilateral vocal cord paralysis. [Comparative Study Research Support, Non-U.S. Gov't]. Laryngoscope, 120(10), 2042-2046. doi: 10.1002/lary.21097

Gliklich, R.E., Glovsky, R.M., \& Montgomery, W.W. (1999). Validation of a voice outcome survey for unilateral vocal cord paralysis. [Clinical Trial Research Support, Non-U.S. Gov't]. Otolaryngol Head Neck Surg, 120(2), 153-158.

Ko, H.C., Lee, L.A., Li, H.Y., \& Fang, T.J. (2009). Etiologic features in patients with unilateral vocal fold paralysis in Taiwan. Chang Gung Med J, 32(3), 290-296.

Lau, D.P., Lee, G.A., Wong, S.M., Lim, V.P., Chan, Y.H., Tan, N.G., Morrison, M.D., et al. (2010). Injection laryngoplasty with hyaluronic acid for unilateral vocal cord paralysis. Randomized controlled trial comparing two different particle sizes. [Comparative Study Randomized Controlled Trial Research Support, Non-U.S. Gov't]. J Voice, 24(1), 113-118. doi: 10.1016/j.jvoice.2008.05.007

Lu JR, T.H., \& Tsai, Y.J. (2003). Assessment of health-related quality of life in Taiwan (I): Development and psychometric testing of SF-36 Taiwan Version. Tai J Pub Health, 22, 501-511.

Monaco, G.N., Brown, T.J., Burgette, R.C., Fargo, K.N., Akst, L.M., Jones, K.J., \& Foecking, E.M. (2013). Electrical stimulation and testosterone enhance recovery from recurrent laryngeal nerve crush. Restor Neurol Neurosci. doi: 10.3233/RNN-130334

Netterville, J.L., Stone, R.E., Luken, E.S., Civantos, F.J., \& Ossoff, R.H. (1993). Silastic medialization and arytenoid adduction: 
The Vanderbilt experience. A review of 116 phonosurgical procedures. Ann Otol Rhinol Laryngol, 102(6), 413-424.

Omori, K., Kacker, A., Slavit, D.H., \& Blaugrund, S.M. (1996). Quantitative videostroboscopic measurement of glottal gap and vocal function: An analysis of thyroplasty type I. [Clinical Trial]. Ann Otol Rhinol Laryngol, 105(4), 280-285.

Pei, Y.C., Fang, T.J., Li, H.Y., \& Wong, A.M.K. (2014). Cricothyroid muscle dysfunction impairs vocal fold vibration in unilateral vocal fold paralysis. Laryngoscope, 124(1), 201-206. doi: Doi 10.1002/Lary.24229

Prendes, B.L., Yung, K.C., Likhterov, I., Schneider, S.L., Al-Jurf, S.A., \& Courey, M.S. (2012). Long-term effects of injection laryngoplasty with a temporary agent on voice quality and vocal fold position. Laryngoscope, 122(10), 2227-2233. doi: 10.1002/lary.23473

Rosenthal, L.H., Benninger, M.S., \& Deeb, R.H. (2007) Vocal fold immobility: A longitudinal analysis of etiology over 20 years. Laryngoscope, 117(10), 1864-1870. doi: 10.1097/MLG.0b013e3180de4d49

Song, P.C., Sung, C.K., \& Franco, R.A., Jr. (2010). Voice outcomes after endoscopic injection laryngoplasty with hyaluronic acid stabilized gel. Laryngoscope, 120 (Suppl 4), S199. doi: 10.1002/lary.21666
Sulica, L. (2008). The natural history of idiopathic unilateral vocal fold paralysis: Evidence and problems. [Review]. Laryngoscope, 118(7), 1303-1307. doi: 10.1097/MLG. Ob013e31816f27ee

Tseng, H.M., L.J., \& Tsai, Y.J. (2003). Assessment of health-related quality of life (II): Norming and validation of SF-36 Taiwan Version. Tai J Pub Health, 22, 512-518.

Wang, L., Rouleau, D.M., \& Beaumont, E. (2013). Most effective adjuvant treatments after surgery in peripheral nerve laceration: Systematic review of the literature on rodent models. [Review]. Restor Neurol Neurosci, 31(3), 253-262. doi:10.3233/RNN120253

Woodson, G.E. (2007). Spontaneous laryngeal reinnervation after recurrent laryngeal or vagus nerve injury. Ann Otol Rhinol Laryngol, 116(1), 57-65.

Yumoto, E., Minoda, R., Hyodo, M., \& Yamagata, T. (2002). Causes of recurrent laryngeal nerve paralysis. Auris Nasus Larynx, 29(1), 41-45.

Yung, K.C., Likhterov, I., \& Courey, M.S. (2011). Effect of temporary vocal fold injection medialization on the rate of permanent medialization laryngoplasty in unilateral vocal fold paralysis patients. [Comparative Study]. Laryngoscope, 121(10), 21912194. doi:10.1002/lary.21965 